\title{
Cigarette smoke exposure disturbed maturation of ovarian follicle and induced abnormal growth of uterus inner wall of female rats \\ Hae-Miru Lee and Kyung-Chul Choi Correspondence
}

Laboratory of Biochemistry and Immunology, College of Veterinary Medicine, Chungbuk National University, Cheongju, Chungbuk 361-763 Republic of Korea

\section{ABSTRACT}

Cigarette smoke (CS) is well known to be very harmful to human body functions such as fertility, reproduction, and Uterus development. To elucidate the effect of CS on women's fertility through maturation follicle and development uterus, more definitely, we examined the histophaological characteristics of the uterus and ovary, this samples received from Korean Conformity Laboratories (KCL), which were obtained from the female rats exposed to the different amounts (low, medium, and high concentrations) of smoke of the standard cigarette (3R4F) for $2 \mathrm{~h} / \mathrm{day}$ and 5 days/week for 28 days according to the OECD guidelines. The animals used for the present study were the spontaneously hypertensive female Wistar Kyoto (WK) rats. We manufactured tissue slides from uterus and ovary samples and evaluated maturation of follicle of ovary and uterus development through $H \& E$ and immunohistochemistry (IHC). As a result, we confirmed that CS impaired maturation of ovary follicle and abnormal uterus development by CS exposure. In IHC analysis on ovary tissues, the expression of PCNA was decreased, The result of the check maturation follicle via a PCNA and H \& E staining confirmed the reduction in follicle at each stage. These changes are particularly shows a significant change in the secondary stage. For uterus, the thickness of inner wall of uterus was decreased by the exposure to CS at low and medium concentrations. In accordance with this result, the expression of PCNA was decreased, but the expression of Bax and CHOP was increased by exposure to CS at low and medium concentrations. However, acute exposure to CS at high level induced the abnormal over-growth of uterus wall. Taken together, the exposure of CS may have a harmful effect on women's fertility and pregnancy by inducing decreased maturation of ovarian follicle and abnormal growth of uterus inner wall.

\section{PURPOSE}

To investigate the histological effects of CS, we examined the effects of CS that impaired maturation follicle and effect on development of Uteri . We confirm that CS have effects to impaired maturation follicle through follicle counts, and development Uteri through changed the PCNA, Bax, CHOP expression levels.

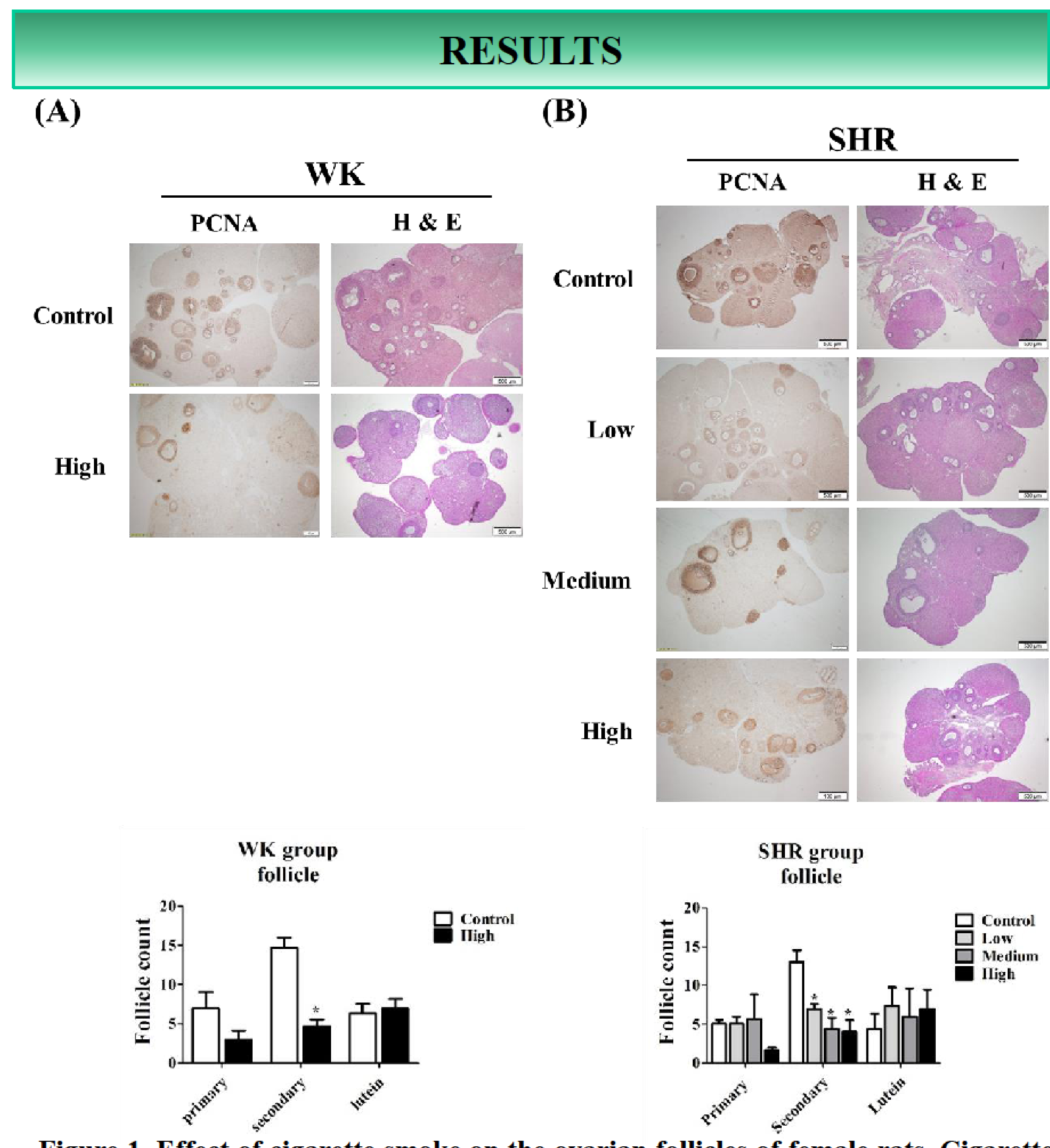

Figure 1. Effect of cigarette smoke on the ovarian follicles of female rats. Cigarette smoke was exposed to (A) WK and (B) SHR female rats under the conditions indicated in Table 1, respectively. At the end of the experiment, ovaries were recovered from the rats and embedded in paraffin. Paraffin blocks were cut into 5 $\mu \mathrm{m}$ thick, and each section was analyzed by IHC to measure PCNA expression as well as by $\mathrm{H} \& \mathrm{E}$ stainging. The numbers of follicles at different stages were counted under the microscope (40x magnification).
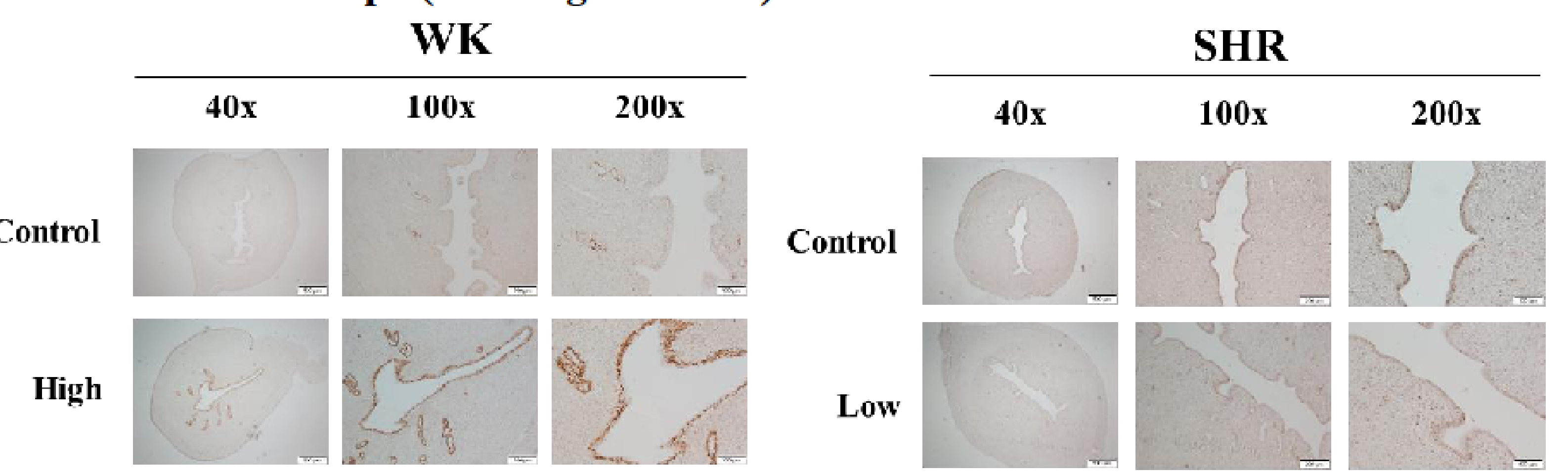

(C)
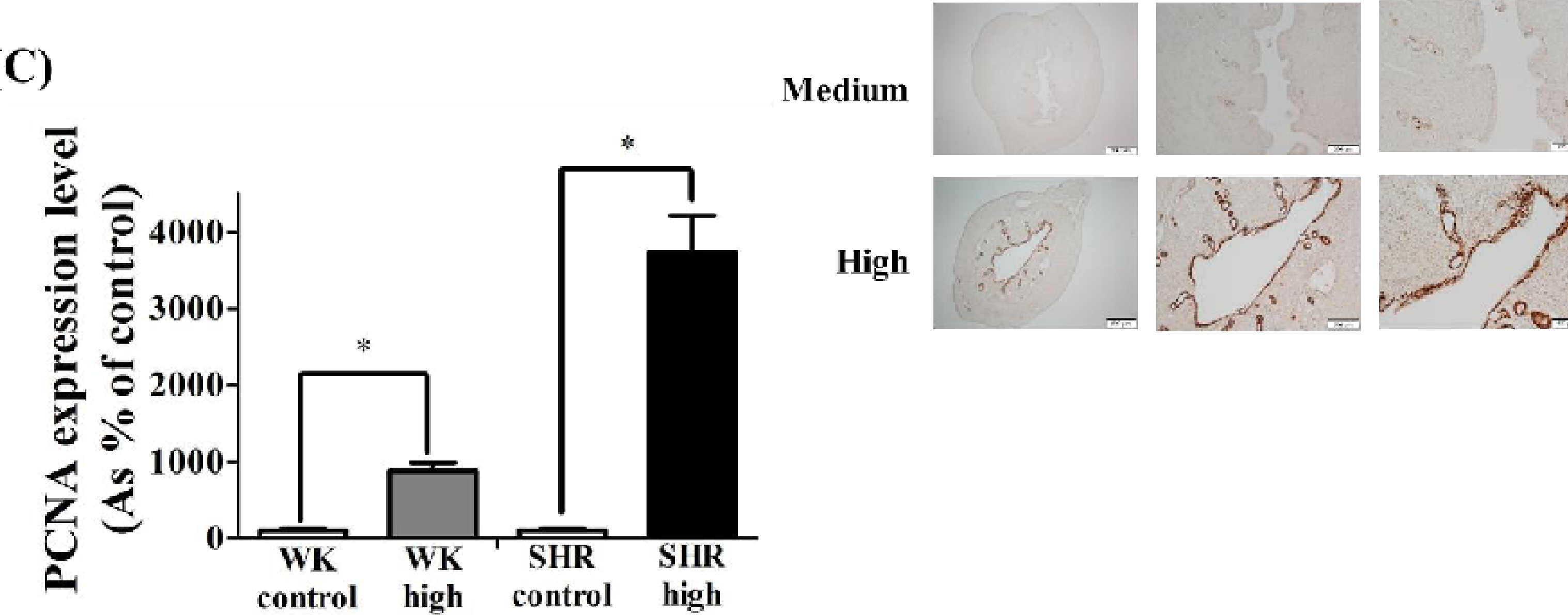

High

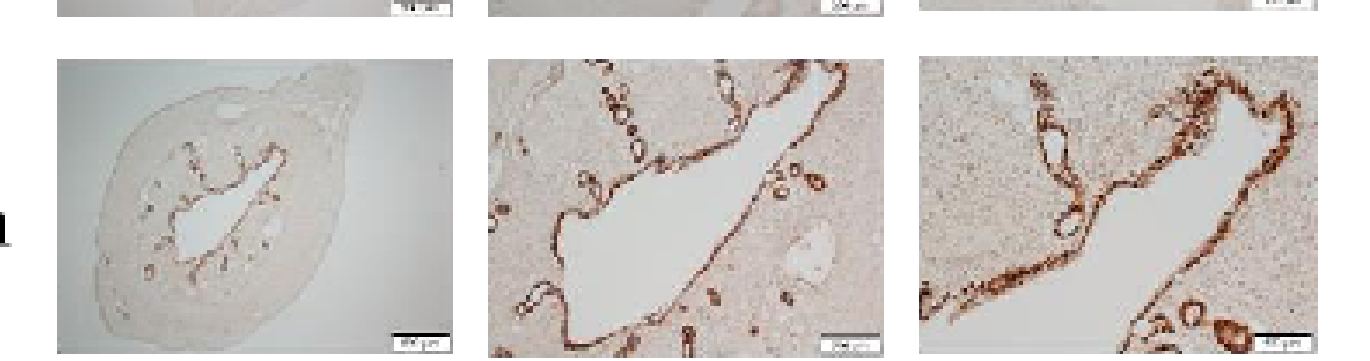
smoke was exposed to (A) WK and (B) SHR rats under the conditions indicated in Table 1, respectively. At the end of the experiment, uteruses were recovered from the rats and embedded in paraffin. Paraffin blocks were cut into $5 \mu \mathrm{m}$ thick, and each section was analyzed by IHC to measure PNCA expression. 40x or 100x or 200x magnification. (C) The PCNA expression levels were quantified by cell sens dimension software (Olympus, Japan).

(A)

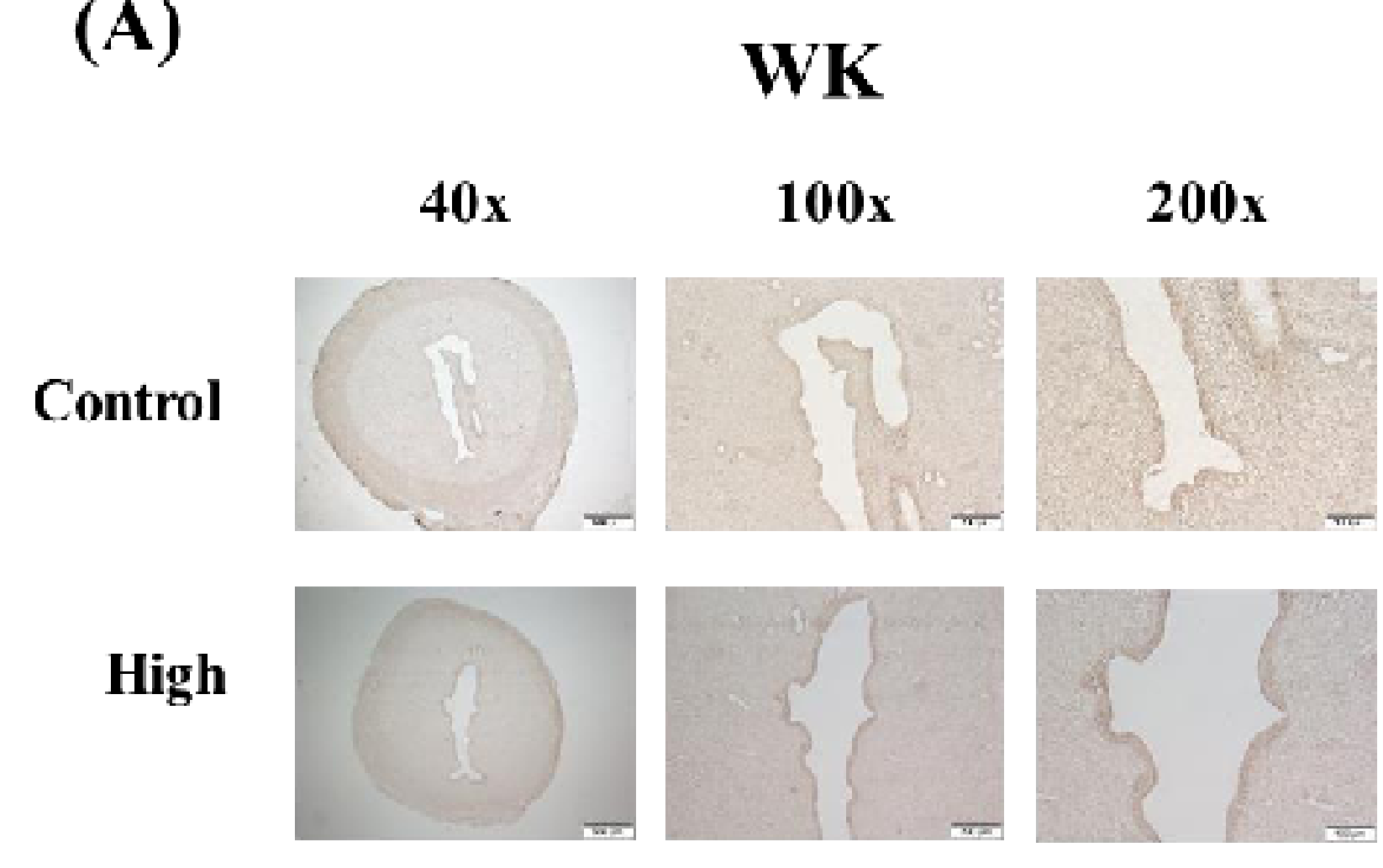

(C)
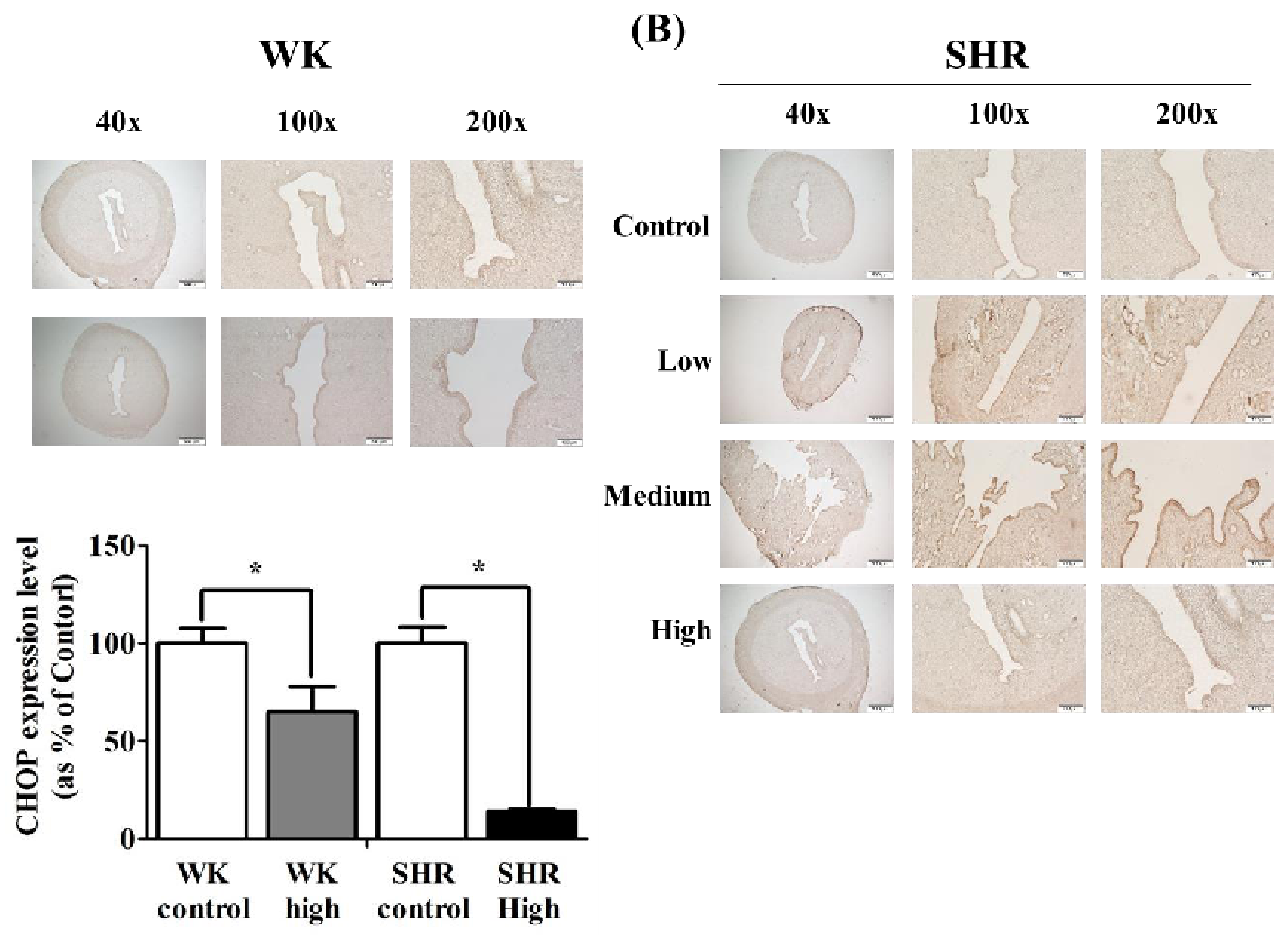

Figure 3. Effect of cigarette smoke on CHOP, an ER-stress marker, in the uteri. Cigarette smoke was exposed to (A) WK and (B) SHR rats under the conditions indicated in Table 1, respectively. At the end of the experiment, uteruses were recovered from the rats and embedded in paraffin. Paraffin blocks were cut into 5 $\mu \mathrm{m}$ thick, and each section was analyzed by IHC to measure CHOP expression. 40x or 100x or 200x magnification. (C) The PCNA expression levels were quantified by cell sens dimension software (Olympus, Japan). follicle and abnormal uterus development.

2. Cigarette smoke induced apoptosis by activations to endoplasmic reticulum stress pathway.

3. Taken together, these results indicate that CS have a abnormal Uteri through promoting apoptosis by actvate ER stress pathway.

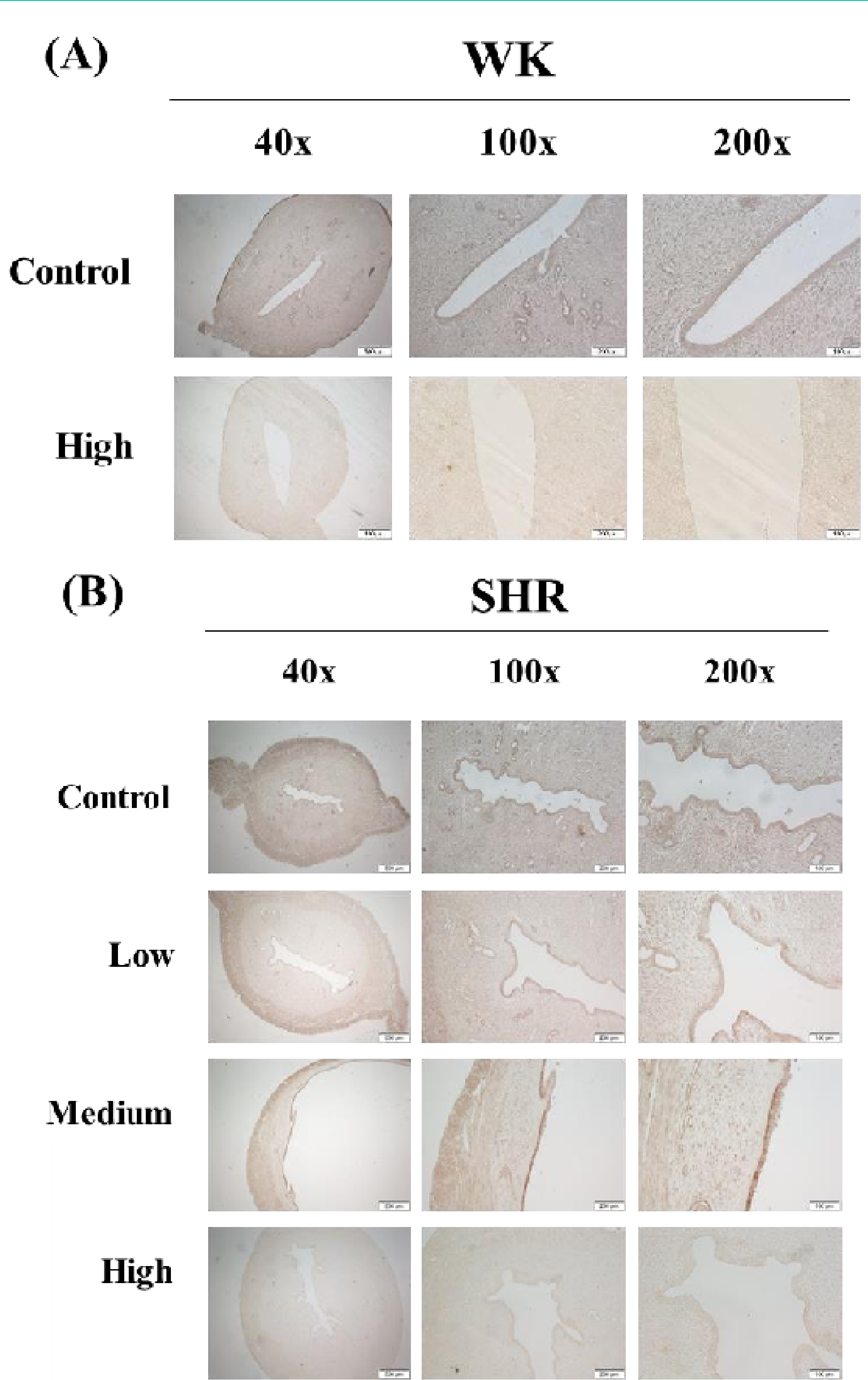

(C)

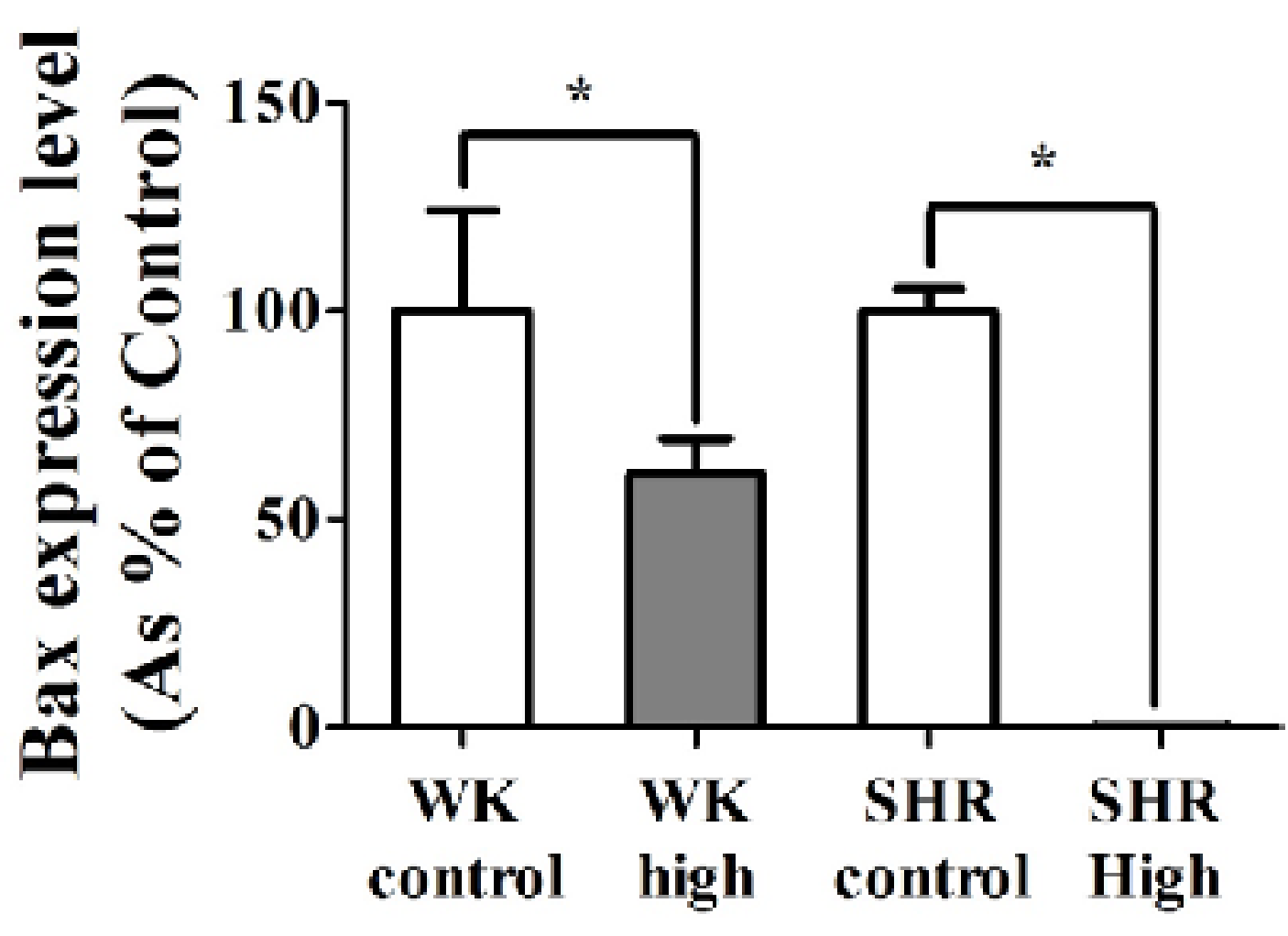

4. Effect of cigarette smoke on Bax, a pro-apoptotic gene, in the uteri. Cigarette smoke was exposed to (A) WK and (B) SHR rats under the conditions
indicated in Table 1, respectively. At the end of the experiment, uteruses were into $5 \mu \mathrm{m}$ thick, and each section was analyzed by IHC to measure Bax (1) (elle

\section{CONCULSION}

4. Taken together, the exposure of CS may have a potential that women's fertility and pregnancy by decreased maturation of ovarian follicle and abnormal growth of uterus inner wall

\section{REFERENCES}

1. Dechanet, C., et al., Effects of cigarette smoking on reproduction. Hum Reprod Update, 2011. 17(1): p. 76-95.

2. Wright, K.P., et al., The effect of female tobacco smoking on IVF outcomes. Hum Reprod, 2006. 21(11): p. 2930-4.

3. Soares, S.R., et al., Cigarette smoking affects uterine receptiveness. Hum Reprod, 2007. 22(2): p. 543-7.

4. Freour, T., et al., Active smoking compromises IVF outcome and affects ovarian reserve. Reprod Biomed Online, 2008. 16(1): p. 96-102.

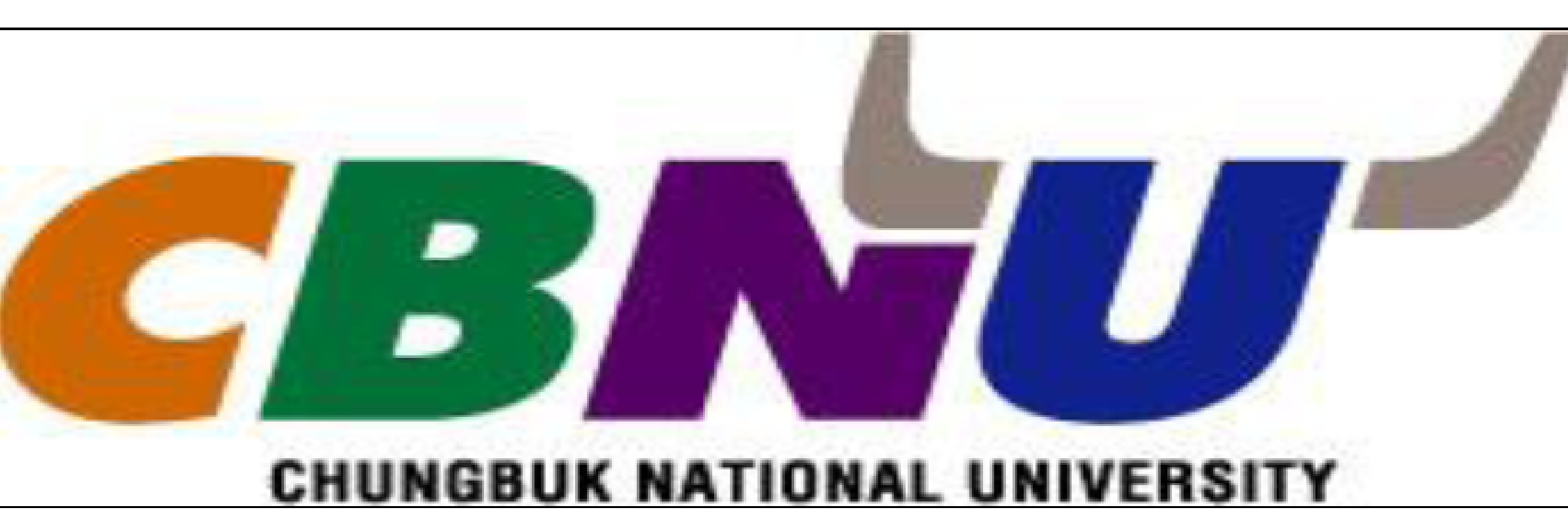

